Int. J. Morphol.,

31(4):1524-1525, 2013.

\title{
Evidence Based Morphology; Quo Vadis?
}

\author{
Morfología Basada en Evidencia; ¿Quo Vadis?
}

\author{
Iván Suazo Galdames*
}

SUAZO, G. I. Evidence Based Morphology; Quo Vadis? Int. J. Morphol., 31(4):1524-1525, 2013.

SUAZO, G. I. Morfología basada en Evidencia; ¿Quo Vadis? Int. J. Morphol., 31(4):1524-1525, 2013.

To the Editor,

Evidence based medicine seeks to determine the best available evidence for problem-solving situations in specific clinical issues (Sackett et al., 2000). However, its tools can be applied to other fields of knowledge where it is necessary to generate opinions, to offer recommendations or to update teaching research and guide this area towards a scientific knowledge.

In 2009, we coined the term evidence-based morphology (Suazo \& Manterola, 2009) so as to highlight a publication which we consider the first systematic review of morphological literature, which in turn, sought to solve structural aspects of the vastus lateralis muscle (Becker et al., 2009). Afterwards, we examined morphological literature and concluded that the majority of the articles and publications concerning morphology correspond to cross sectional studies which can be meta-analyzed when they count with the adequate morphological quality (Suazo \& Materola, 2010). With the objective of evaluating if it was possible to answer a clinical-related question, we systematically reviewed the literature related to the percentage of insertion in the superior head of the lateral pterygoid muscle in the TMJ disk. We found that the studies were not at all comparable (Tapia et al., 2011).

During 2013, the focus still lies on case reports, cross sectional studies and narrative reviews even though we identified two articles which have been satisfactorily published and that incorporate a systematic review and metaanalysis of morphological literature for solving anatomical and/or clinical-surgical questions (Raikos \& Paraskevas, 2013; Yammine, 2013). This supposes that medical tools based on evidence are now being considered by researchers in morphological sciences as valid instruments for problemsolving situations related to the field.

KEY WORDS: Evidence based morphology; Evidence based medicine; Literature review.

\section{Señor Editor}

La medicina basada en evidencia busca determinar la mejor evidencia disponible para resolver problemas clínicos específicos (Sackett et al., 2000), sin embargo sus herramientas pueden ser aplicadas a otras áreas del conocimiento donde sea necesario general opinión, entregar recomendaciones o simplemente con fines docentes para entregar un conocimiento actualizado y basado en conocimiento científico.

El 2009 acuñamos el término Morfología basada en evidencia (Suazo \& Manterola, 2009) para destacar una publicación, que consideramos la primera revisión sistemática de la literatura morfológica, ésta buscaba resolver aspectos de la estructura del músculo vasto lateral (Becker et al., 2009). Luego analizamos la literatura morfológica concluyendo que la mayor parte de los artículos publicados en morfología corresponden a cortes transversales que pueden ser meta-analizados cuando cuentan con calidad metodológica suficiente (Suazo \& Manterola, 2010). Posteriormente, con la finalidad de evaluar si era posible entonces, responder a una pregunta de interés clínico, revisamos sistemáticamente la literatura referida al porcentaje de inserción de la cabeza superior del músculo pterigoideo lateral en el disco de la ATM humana, encontrando que los estudios eran difícilmente comparables (Tapia et al., 2011).

Al año 2013 el predominio sigue estando en las los reportes de caso, cortes transversales y revisiones narrativas, aunque vemos con satisfacción que han sido publicados dos artículos que incorporan una revisión sistemática y metaanálisis de literatura morfológica para resolver cuestionamientos anatómicos y de interés clínico-quirúrgico (Raikos \& Paraskevas, 2013; Yammine, 2013), lo que supone que las herramientas de la medicina basada en evidencia comienzan a ser consideradas por los investigadores de las ciencias morfológicas como instrumentos válidos para resolver problemas de la disciplina.

PALABRAS CLAVE: Morfología basada en evidencia; Medicina basada en evidencia; Revisión de la literatura. 


\section{REFERENCES}

Becker, I.; Woodley, S. J. \& Baxter, G. D. Gross morphology of the vastus lateralis muscle: An anatomical review. Clin. Anat., 22(4):436-50, 2009.

Raikos, A. \& Paraskevas, G. K. The thyroid foramen: a systematic review and surgical considerations. Clin. Anat., 26(6):700-8, 2013.

Sackett, D. L.; Strauss, S. E.; Richardson, W. S.; Rosenberg, W. \& Haynes, R. B. Evidence-based medicine: how to practice and teach EBM. 2nd ed. London, Churchill-Livingstone, 2000

Suazo, G. I. \& Manterola, D. C. The beginning of the evidencebased morphology. Clin. Anat., 22(6):783, 2009.

Suazo, G. I. \& Manterola, D. C. What is published in Human Morphology? Types of designs and Levels of evidence. Int. J. Morphol., 28(2):461-70, 2010.

Tapia, J. C.; Cantín, M.; Zavando, D. \& Suazo, G. I. Percentage of Lateral Pterygoid Muscle Inserted in the Disc of the Human Temporomandibular Joint. Int. J. Morphol., 29(3):965-70, 2011.

Yammine, K. Clinical prevalence of palmaris longus agenesis: a systematic review and meta-analysis. Clin. Anat., 26(6):70918, 2013.
Correspondence to:

Prof. Dr. Iván Suazo G.

Dirección de Investigación y Postgrado

Universidad Autónoma de Chile

Carlos Antúnez 1920, Providencia, Santiago

CHILE

Email: ivan.suazo@uautonoma.cl

Received: 12-08-2013

Accepted: 08-11-2013 